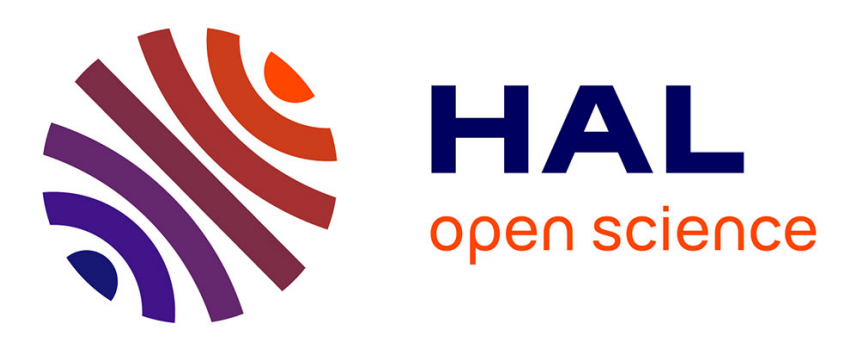

\title{
La vulnérabilité, un concept central de l'analyse des risques urbains en lien avec le changement climatique
}

Béatrice Quenault

\section{To cite this version:}

Béatrice Quenault. La vulnérabilité, un concept central de l'analyse des risques urbains en lien avec le changement climatique. Les Annales de la Recherche Urbaine, 2015, septembre (110). hal-01645003

\section{HAL Id: hal-01645003 \\ https://hal.univ-rennes2.fr/hal-01645003}

Submitted on 22 Nov 2017

HAL is a multi-disciplinary open access archive for the deposit and dissemination of scientific research documents, whether they are published or not. The documents may come from teaching and research institutions in France or abroad, or from public or private research centers.
L'archive ouverte pluridisciplinaire HAL, est destinée au dépôt et à la diffusion de documents scientifiques de niveau recherche, publiés ou non, émanant des établissements d'enseignement et de recherche français ou étrangers, des laboratoires publics ou privés. 


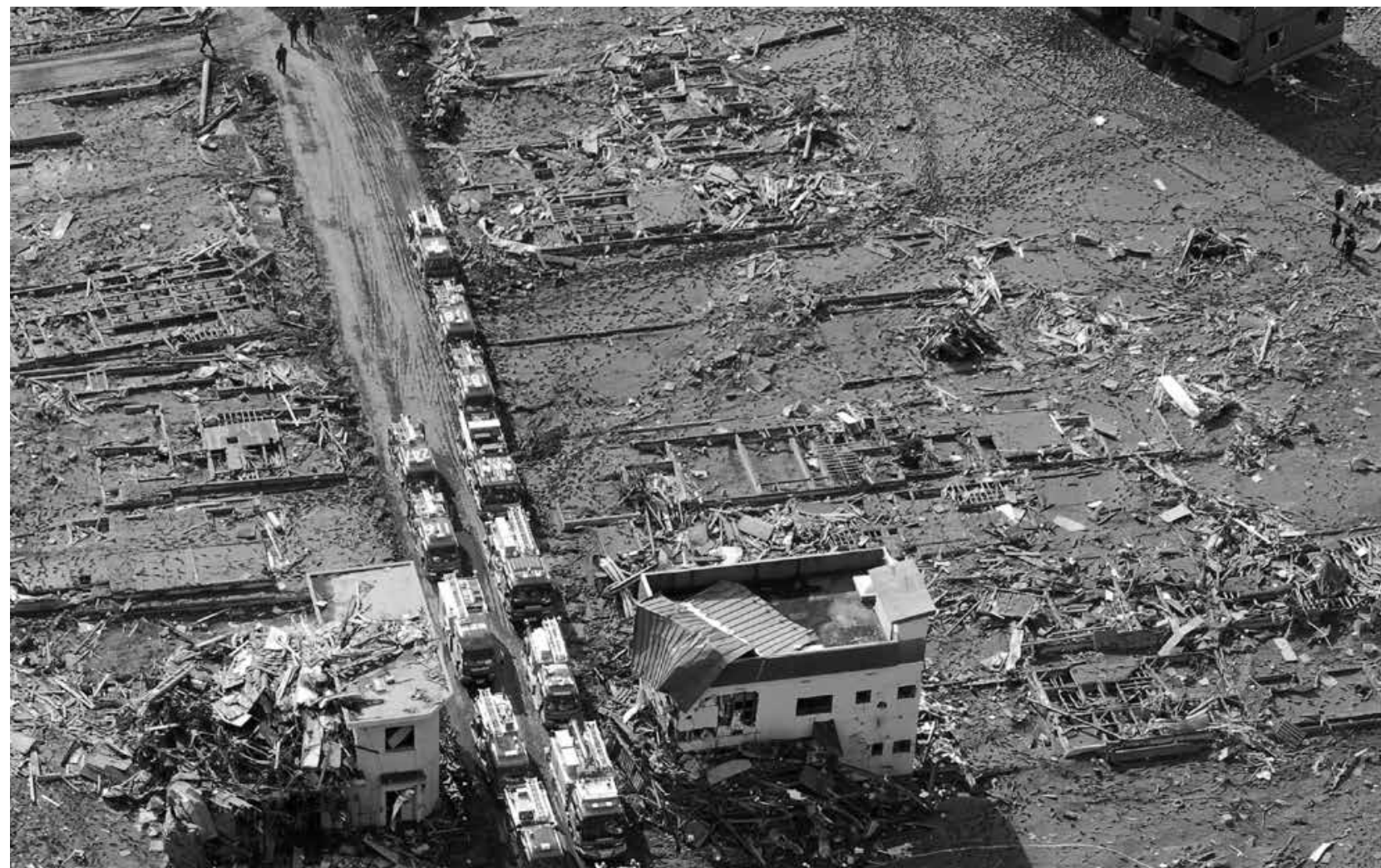

2. 15.1 .5

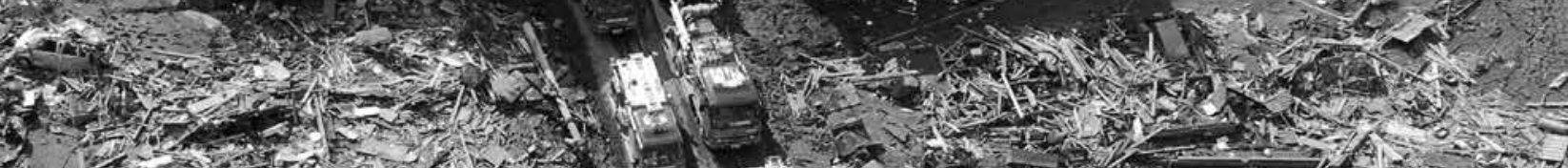

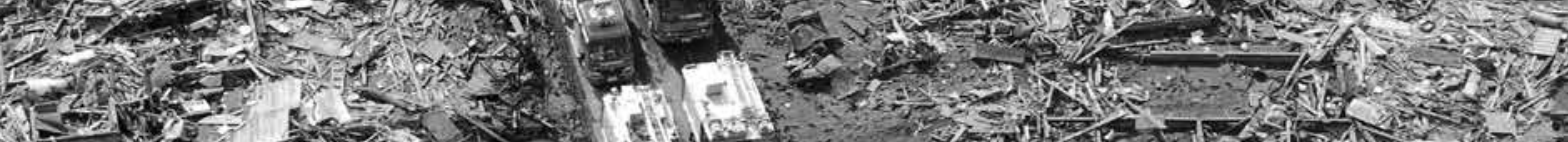

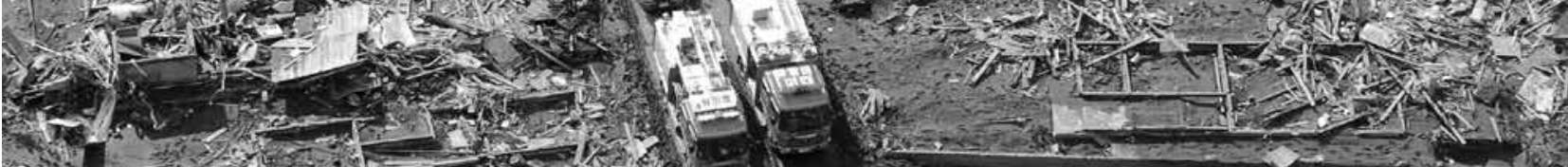
364 (1)

S.r.

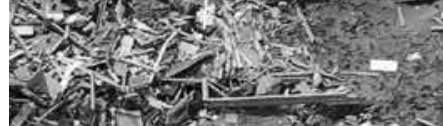

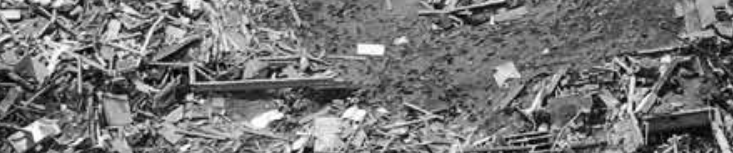

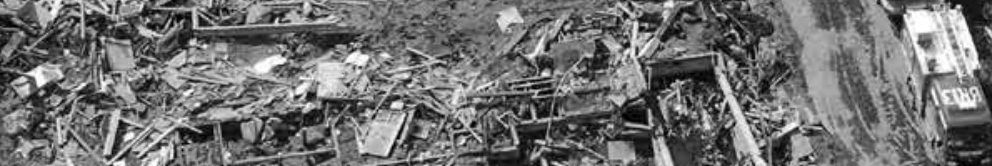

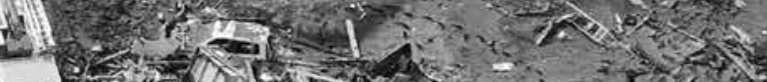

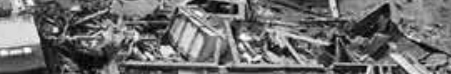
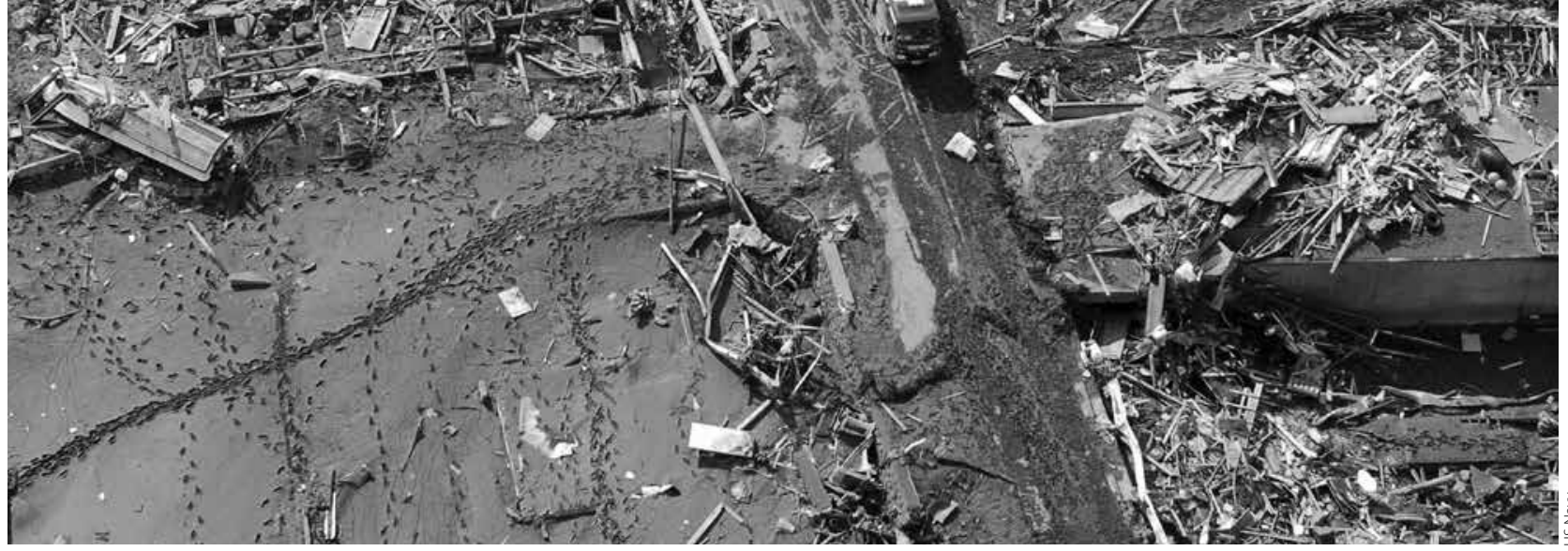


\section{La vulnérabilité, un concept central de l'analyse des risques urbains en lien avec le changement climatique}

\section{Béatrice Quenault}

Dans la seconde moitié du XX ${ }^{\mathrm{e}}$ siècle, les villes du monde entier ont connu une forte augmentation des épisodes dramatiques entraînant des pertes humaines et des dommages parfois exorbitants, les aléas climatiques ${ }^{1}$ ayant joué le rôle de déclencheurs pour la majorité d'entre eux. Les catastrophes " naturelles ${ }^{2}$ » liées aux événements climatiques extrêmes ${ }^{3}$

1. Si le lien entre le changement climatique et ces événements extrêmes dommageables apparaît a priori évident (par exemple, la montée du niveau des mers liée au changement climatique rendra les villes côtières plus vulnérables aux événements extrêmes que représentent les fortes tempêtes), il est statistiquement difficile d'établir des relations de cause à effet entre des événements isolés et des tendances à long terme liées au changement climatique en cours. En revanche, ces épisodes dramatiques attestent bel et bien de la vulnérabilité des grandes villes et des établissements humains plus petits à ces aléas.

2. On parle de catastrophe « naturelle » pour désigner « un événement naturel désastreux et destructeur, susceptible de modifier plus ou moins profondément, et sur un territoire plus ou moins étendu, l'environnement et les conditions de vie des groupes humains, voire même de détruire à la fois leurs vies, leurs biens et leurs installations. Quand les destructions sont massives, on parle de cataclysme » (Dunglas, 2005, p. 91). Il ne faut toutefois pas se méprendre sur le terme « naturel ", dans la mesure où en réalité les catastrophes ont toutes nécessairement une dimension sociale (un événement extrême se produisant dans un désert ne provoquera pas de catastrophe), et où les causes premières des aléas dits « naturels » sont le plus souvent anthropiques, comme dans le cas du changement climatique et de ses impacts (intensification ou multiplication des événements extrêmes, par exemple)

3. L'événement météorologique extrême renvoie à des épisodes considérés comme exceptionnels (i.e. intenses et peu probables) par les valeurs inhabituelles atteintes par un ou plusieurs paramètres (température, vent, pluie, etc.) sur un territoire donné. Les événements extrêmes sont caractérisés par une faible probabilité d'occurrence mais, lorsqu'ils se produisent, ils entraînent, compte tenu de leur intensité, de fortes conséquences sur les villes en particulier. Le principal problème avec le changement climatique ne réside pas tant dans l'intensification de ces perturbations et de leur occurrence, estimée « très probable » par les modèles climatiques globaux (Giec, 2007), que dans l'aggravation de leurs impacts compte tenu notamment des perspectives démographiques et urbanistiques mondiales qui augmentent la vulnérabilité des villes. comptent ainsi parmi les désastres qui frappent aujourd'hui le plus les esprits : sur les 8500 catastrophes «naturelles » environ survenues entre 1970 et 2009, les vingt-cinq les plus coûteuses se sont produites après 1987, et parmi elles, vingttrois étaient liées aux conditions climatiques ; ces dernières auraient ôté la vie à près de deux millions de personnes, et causé des dégâts estimés à 1500 milliards de dollars (Cred, 2010). En 2013, sur les 330 événements répertoriés par la base internationale EM-DAT, qui ont fait 21600 morts, 96,5 millions de victimes et 118,6 milliards de dommages matériels, 90 \% d'entre eux étaient liés à des aléas hydrométéorologiques ou climatiques et seulement $10 \%$ à des aléas géologiques (Cred, 2014). L'aggravation des impacts dus aux aléas hydrométéorologiques ou climatiques est consécutive à la pression anthropique croissante que l'urbain exerce sur les milieux et aux modalités de l'urbanisation elle-même qui ont fait des agglomérations de véritables points névralgiques (Quenault, 2013a).

Ces catastrophes ont certes dramatiquement rappelé aux pays (développés en particulier) l'existence de multiples menaces, mais elles ont surtout souligné leur fragilité face à de tels épisodes, alors même qu'ils s'estimaient protégés grâce aux progrès technologiques et scientifiques accomplis en matière de prévention. L'occurrence de ces événements catastrophiques a été interprétée non pas tant comme la résurgence du « risque » que comme l'expression de la «vulnérabilité » des sociétés concernées. La notion de "vulnérabilité ", avec ses corrélats de risque, de capacité d'adaptation, et de résilience, a émergé au niveau international dans les années 1990 et dans le champ des sciences humaines et sociales au cours des années 1980. Elle est aujourd'hui un concept central de l'analyse des risques « naturels » en milieu urbain,

Les Annales de la recherche urbaine $n^{\circ} 110,2015$, pp. 138-151, (C) MEDDE, Puca. 
en particulier en lien avec le changement climatique et la problématique du développement durable (Quenault, 2015). Les approches ont ainsi progressivement opéré un changement de perspective, en résonance avec l'accroissement des risques et des catastrophes en milieu urbain, signes d'une société plus vulnérable : d'un regard centré principalement sur les aléas, on est passé à une vision qui s'intéresse de plus en plus à la vulnérabilité, celle-ci ayant elle-même connu un élargissement au fil des travaux qui lui ont été consacrés. Pour autant, dans la littérature sur les risques naturels/anthropiques, le concept de vulnérabilité urbaine reste encore largement à définir et à préciser (Quenault et al., 2011).

Dans ce contexte, l'objectif de cet article est double : il s'agit, en premier lieu, de souligner comment s'est opérée la reconnaissance institutionnelle et académique croissante du concept de vulnérabilité pour analyser les risques urbains, notamment en lien avec le changement climatique ; et en second lieu, de montrer que ce concept polysémique et multidisciplinaire se trouve mobilisé suivant des acceptions très diverses et souvent décliné selon différents champs ou dimensions, au point que l'on peut se demander si la notion ne souffrirait pas d'un trop-plein sémantique pour analyser la dynamique complexe des vulnérabilités urbaines face aux risques climatiques.

\section{Reconnaissance croissante du concept de vulnérabilité dans l'analyse et la gestion des risques}

Au sein du couple aléa-vulnérabilité qui caractérise le risque, la seconde composante est longtemps restée peu explorée, les recherches et analyses se concentrant quasi exclusivement sur l'aléa, ainsi que le déploraient déjà Jacques Theys et Jean-Louis Fabiani dans leur ouvrage précurseur, Les travaux sur la vulnérabilité restent de loin les parents pauvres des recherches sur les risques et la sécurité $(1987$, p. 21) ; constat réaffirmé quelques vingt ans plus tard par Claude Gilbert qui, soulignant l'importance de la dimension culturelle et sociale de la genèse des désastres et catastrophes, déclare : «Il est peut-être temps de prendre au sérieux la vulnérabilité et d'engager à son sujet des recherches tout aussi longues et complexes que celles concernant les aléas »(Gilbert, 2006, p. 120). Ces exhortations sont en phase avec la tendance, déjà ancienne et en fort développement, à attirer l'attention sur l'étude de la vulnérabilité (D'Ercole, 1994 ; Becerra et Peltier, 2009 ; D’Ercole et Metzger, 2009).

\section{Un cadre analytique commun pour conceptualiser les interactions urbaines et écologiques globales}

En France, la thématique de la vulnérabilité arrive vers le milieu des années 1980 avec la publication de La société vulnérable (Fabiani et Theys, 1987), qui fait écho à La société du risque du sociologue allemand Ulrich Beck (1986).
Dans leur ouvrage, où ils s'interrogent sur la dangerosité des sociétés contemporaines comparée à celles du passé, Fabiani et Theys mettent en lumière un basculement de la problématique du risque : si les dangers ne sont ni plus nombreux, ni plus intenses qu'auparavant, en revanche, la vulnérabilité des sociétés frappées par les aléas a progressé. On glisse ainsi d'une société du risque à une société vulnérable, au sein de laquelle le sentiment d'insécurité s'accroît alors même qu'un dispositif sécuritaire sans précédent a été déployé pour maîtriser les risques. La plupart des organisations et instances internationales ont dès lors adopté le concept de vulnérabilité dans leurs travaux et rapports, tandis que plusieurs disciplines y ont eu recours pour en faire un cadre analytique commun. Ce concept a l'avantage de pouvoir être utilisé comme un cadre général pour conceptualiser et analyser les relations causales entre la nature des risques, les réponses qui y sont apportées, et les résultats ou impacts qui découlent d'événements ou de processus risqués. La vulnérabilité peut être en effet un cadre pertinent pour mieux appréhender les interactions entre les processus écologiques relatifs à la biosphère (tels que les changements globaux) et les processus sociaux et politiques associés au développement urbain (Quenault et al., 2011).

Pour les chercheurs travaillant sur les risques (anthropologues, économistes, géographes, juristes, sociologues, etc.), l'approche en termes de vulnérabilité a permis de prolonger les réflexions qui, depuis les années 1980, ont conduit à considérer les catastrophes " naturelles » non comme des ruptures de la normalité ou des dysfonctionnements de l'ordre social, mais comme des produits de cet ordre (Hewitt, 1983). Ce faisant les travaux se sont orientés non plus tant sur les diverses manières qu'avaient les sociétés touchées d'affronter les catastrophes et d'en contrebalancer les effets que sur les conditions sociales, politiques, économiques et culturelles contribuant à produire les désastres. Cela transparaît dans la définition qu'en donne D’Ercole (1994, p. 88-89) : « La vulnérabilité apparaît comme la propension d'une société donnée à subir des dommages en cas de manifestation d'un phénomène naturel ou anthropique. Cette propension varie selon le poids de certains facteurs qu'il est nécessaire d'identifier et d'analyser car ils induisent un certain type de réponse de la société. » Dès lors, la vulnérabilité ne désigne plus uniquement l'ensemble des dommages provoqués par la réalisation d'un aléa, mais également l'ensemble des facteurs sociétaux qui rendent une société plus ou moins fragile face à l'occurrence d'un tel événement.

À la recherche des facteurs de vulnérabilité et des moyens de la réduire

La littérature scientifique préoccupée par les risques « naturels » en milieu urbain a connu de substantiels développements au cours des décennies écoulées : les ouvrages de protection relevant d'une approche aléa- 


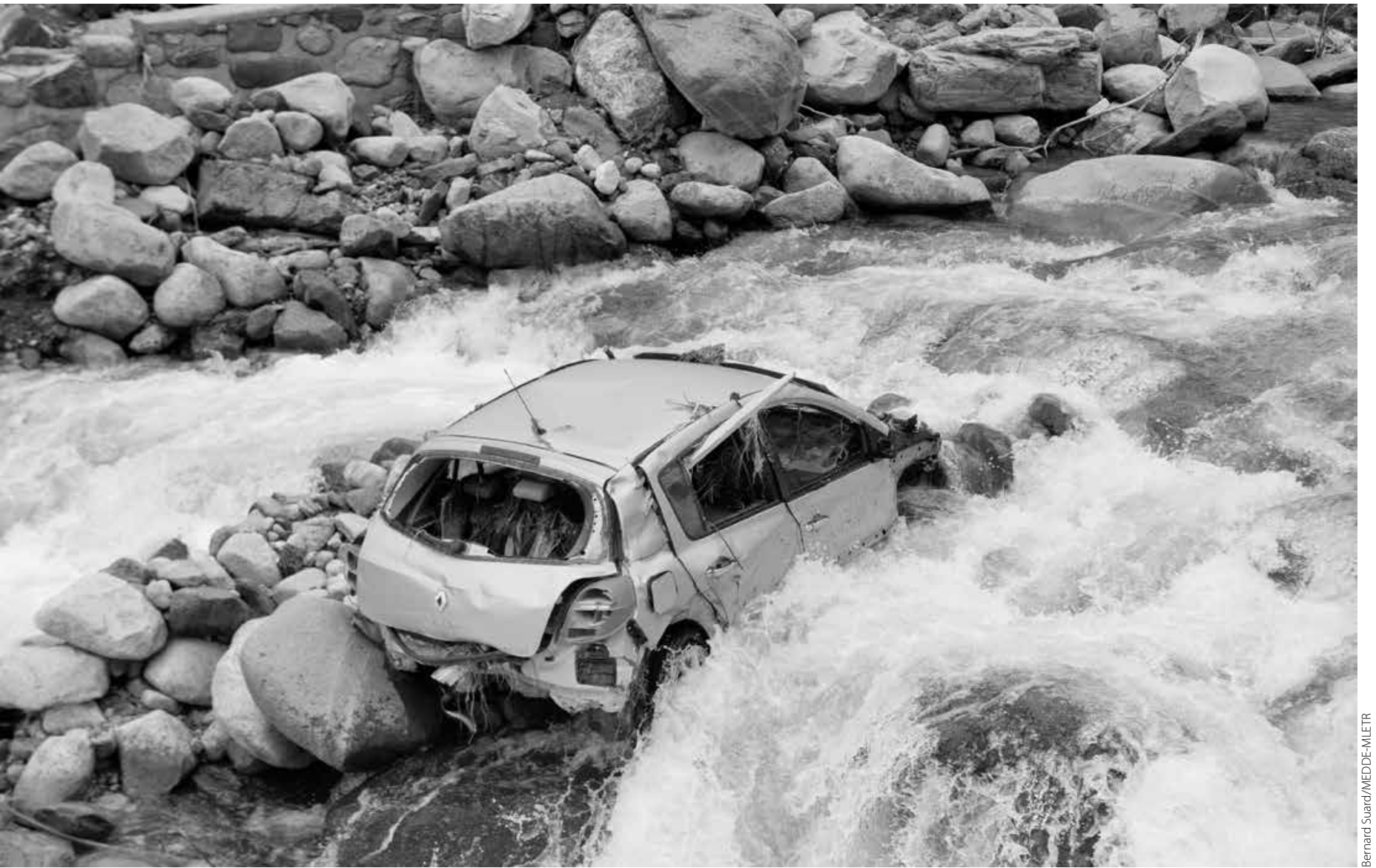

centrée de la gestion des risques ayant montré leurs limites dans les espaces urbanisés - quand ils n'ont pas contribué à augmenter les risques -, les préoccupations se sont progressivement reportées sur la compréhension de ce qui fait la vulnérabilité urbaine. De ce point de vue, la littérature anglosaxonne offre depuis plusieurs années des voies d'analyse intéressantes notamment avec les travaux fondateurs de Blaikie et al. (1994).

Dans le prolongement de ces analyses, lors de la Décennie internationale pour la réduction des catastrophes naturelles (DIRCN) (International Decade for Natural Disaster Reduction ou IDNDR), instaurée en 1990 sous l'égide des Nations unies, va s'ouvrir pour la première fois uwn grand forum de discussion entre les différents acteurs concernés par les catastrophes naturelles. Une inflexion majeure se produit alors, l'attention se détournant quelque peu de l'aléa lui-même pour se focaliser davantage sur la société et la réduction des « vulnérabilités ». La conférence de Yokohama, en 1994, qui coïncide avec la parution de l'ouvrage dirigé par Blaikie et ses collègues, At Risk: Natural Hazards, People's Vulnerability, est en effet la première à consacrer une session entière à l'évaluation de la vulnérabilité sociale et à placer la « réduction de la vulnérabilité » à l'ordre du jour de la DIRCN et au centre des recherches sur les catastrophes naturelles.
Le concept de vulnérabilité connaît alors un succès d'autant plus rapide que l'affirmation du développement durable comme cadre d'action et de réflexion pour le XXI ${ }^{\mathrm{e}}$ siècle en accélère la promotion à l'issue du $3^{\mathrm{e}}$ Sommet de la terre à Rio de Janeiro (1992). À partir de là, la vulnérabilité liée au risque va apparaître comme « une mesure de la non-durabilité »(Gray et Wiedermann, 1997, p. 6). À la lumière de la fréquence croissante des catastrophes en milieu urbain et de la dégradation écologique continue de la planète, appréhender la vulnérabilité dans toute sa complexité est perçu comme une tâche cruciale dès lors que l'objectif de la réflexion comme de l'action est d'aider à opérer la transition vers un monde plus soutenable grâce à la « fabrication » de sociétés résilientes ${ }^{4}$ aux catastrophes (Quenault, 2012).

4. La résilience est souvent conçue comme la « face opposée » de la vulnérabilité, son pendant positif. L'attribution d'une connotation positive à la résilience est liée à la volonté d'en assurer l'applicabilité et l'acceptabilité politique : alors que la vulnérabilité renvoie à une fragilité ou à une incapacité, la résilience est au contraire érigée en propriété désirable d'un système vers laquelle la gestion des risques ou la planification urbaine devraient tendre. Or, une telle vision, trop simpliste, passe selon nous à côté des liens complexes de la vulnérabilité et de la résilience (Quenault, 2013b). 
Cette perception contraste fortement avec les recherches et les stratégies du passé qui ont souvent été purement aléa-centrées. L'aptitude à pouvoir apprécier les vulnérabilités des sociétés humaines est perçue de manière croissante comme un pas essentiel franchi vers une réduction des risques effective et la promotion d'une culture de la résilience ${ }^{5}$ aux désastres. Plutôt que de définir principalement les catastrophes comme des occurrences physiques, requérant largement des solutions technologiques, s'impose l'idée qu'il convient de les concevoir comme le résultat d'interactions complexes entre un événement physique potentiellement dangereux (ici les aléas liés au changement et à la variabilité climatiques) et les vulnérabilités d'une société déterminées par les comportements et les choix humains. Vues sous cet éclairage, les catastrophes naturelles sont perçues et comprises comme des désastres "non naturels » invitant à passer d'une focalisation du regard sur les risques et leur quantification à une attention toute particulière accordée à l'identification, l'évaluation et le classement des différentes vulnérabilités. Face aux multiples acceptions et au flou du concept, cette tâche s'avère pour le moins délicate à mener à bien.

\section{Un concept polysémique et multidisciplinaire souffrant d'un trop-plein sémantique}

Lorsque l'on cherche à étudier la vulnérabilité, on se heurte d'emblée à une "profusion de définitions » (Pigeon, 2005, p. 5), qui finissent par renvoyer à des objets et à des préoccupations très différents. Face à la polysémie dans l'emploi du terme de vulnérabilité, dont Theys et Fabiani (1987) ont souligné qu'il souffrait « d'un tropplein sémantique puisqu'il évoque aussi bien la dépendance ou la fragilité, la centralité, l'absence de régulation efficace, le gigantisme ou la faible résilience », il semble essentiel de pouvoir en clarifier les différentes acceptions. Toutefois, avant de retracer les significations et les emplois du terme de vulnérabilité dans le langage scientifique des risques « naturels » et du changement climatique (comme risque anthropogénique), il convient d'en rappeler l'usage dans le langage courant dans la mesure où l'emploi qui en est fait dans le langage académique en découle.

5. La résilience, qui s'entend comme la capacité d'un système à surmonter un choc ou une perturbation majeure et à se « reconstruire en mieux ", revêt dans son acception contemporaine une double dimension : sa dimension physique ou réactive renvoie à la « capacité à faire face » à un impact donné (comme l'aptitude d'une maison à résister à des vents violents ou la santé physique d'un individu pour survivre à une vague de chaleur); sa dimension sociale ou dynamique (proactive) reflète la « capacité de réponse » face à un choc (capacité d'un territoire urbain, d'une communauté, d'un ménage ou d'un individu à récupérer ou à se reconstruire grâce à des capacités adaptatives liées à l'organisation sociale, économique ou institutionnelle) (Quenault, 2013b).
La vulnérabilité dans le langage courant

D’un point de vue étymologique, le mot «vulnérabilité » vient du bas latin vulnerabilis, lui-même construit à partir du verbe vulnerare, "infliger une blessure à, blesser ", et du suffixe abilis, " qui peut être, enclin à ", suggérant ainsi que le fait d' "être blessé » puisse se produire (possibilité et non nécessité) (Adam, 2008). Dans le langage courant, la vulnérabilité est le caractère de ce qui est vulnérable, c'est-à-dire de ce « qui peut être blessé, frappé par un mal physique » ou de ce qui, au plan moral, « peut être facilement atteint, se défend mal » (Petit Robert, 1991, p. 2122), l'adjectif «vulnérable » étant le plus souvent attribué à une personne ou un objet, désigné sous le vocable général d'« entité ». En d'autres termes, dans le langage courant, la vulnérabilité signifie qu'un dommage est possible dans le futur et, par extension, est synonyme de fragilité face à une menace.

De cette acception, il ressort, en premier lieu, un lien inextricable de la notion de vulnérabilité avec celle d'incertitude : une entité est vulnérable (maintenant) s'il existe une possibilité qu'elle soit « attaquée » ou «blessée » (plus tard) ${ }^{6}$. Ce concept a donc une qualité prédictive en situation d'incertitude : "La vulnérabilité est un terme hypothétique et prédictif »(Blaikie et al., 19947), ou comme l'expriment Calvo et Dercon (2005), « la vulnérabilité est utilisée comme une magnitude de la menace [...], mesurée ex ante, avant que le voile de l'incertitude n'ait été soulevé ${ }^{8} »$. Toutefois, l'incertitude n'est pas un simple effet secondaire résultant de " notre inaptitude à voir le futur ", mais une caractéristique inhérente au concept de vulnérabilité, introduite par l'idée de « possibilité » (Adam, 2008, p. 17). De l'emploi du terme dans le langage courant, il ressort en second lieu que le nom abstrait "vulnérabilité », construit en ajoutant le suffixe -ité, décrit une propriété qui admet des degrés. Des formulations du type «telle personne est plus vulnérable que telle autre » sont fréquemment usitées, révélant par là-même la propriété grammaticale de comparaison de l'adjectif vulnérable, ce qui signifie que le degré auquel le modificatif modifie son complément peut être distingué. La notion de vulnérabilité, utilisée pour désigner un état de fragilité, une propension à subir des dommages, exprime donc la plus ou moins grande susceptibilité d'une personne ou d'un bien d'être à la merci de la moindre atteinte, blessure,

6. On retrouve d'ailleurs l'idée de blessure dans les termes de dommages et de pertes qui interviennent fréquemment dans les définitions scientifiques de la vulnérabilité.

7. Dans l'original : "Vulnerability is a hypothetical and predictive term » (Blaikie et al., 1994).

8. Dans l'original : "Vulnerability is used as the magnitude of the threat [...], measured ex ante, before the veil of uncertainty has been lifted »(Calvo et Dercon, 2005). 
attaque, ou, au contraire, de leur capacité plus ou moins forte à résister face à un risque donné. Deux « primitifs » de la notion peuvent ainsi être identifiés : l'entité dans un état donné à un moment du temps et son évolution future incertaine et la notion d'effets négatifs ou de dommages. Toutefois, lorsque le terme de vulnérabilité est employé dans le langage courant, les situations futures possibles ne sont que rarement explicitées pas plus que n’est spécifiée l'évaluation des dommages (Adam, 2008).

\section{La triple acception de la vulnérabilité dans le champ} des risques naturels

La terminologie relative à la vulnérabilité en tant que terme technique dans le champ des risques naturels, et plus spécifiquement dans celui des changements globaux, est très abondante (Brooks, 2003). Des articles consacrés à une revue de la littérature sur le sujet (Adger, 2006 ; O’Brien et al., 2007) ont recensé plus d'une trentaine de définitions de la vulnérabilité ${ }^{10}$. À première vue, les définitions académiques semblent beaucoup plus complexes que celles du langage courant, car elles mobilisent d'autres concepts tels que ceux d'impacts, de danger, de risque, de capacité à faire face ou à s'adapter, ou encore de résilience, autant de termes qui nécessitent eux-mêmes d'être définis. Cependant, les concepts scientifiques « collent» le plus souvent à la structure de la définition «basique » ou courante de la vulnérabilité. Les deux « primitifs » de la vulnérabilité précédemment identifiés, le futur incertain et les dommages, font partie des éléments récurrents des définitions scientifiques qui peuvent être classés suivant neuf «thèmes » (propriété graduée, dimensions, entité vulnérable, futur incertain, risque, action, dommage, cause du dommage et « autres ») (Adam, 2008).

Pour réduire le foisonnement des définitions académiques existantes, on peut les regrouper en trois groupes distincts : premièrement, les définitions qui mettent l'accent sur les caractéristiques ou l'étiologie de l'aléa et les pertes associées avec sa localisation; deuxièmement, celles qui perçoivent l'événement comme un construit social plutôt que comme une condition biophysique ; et enfin, celles de nature synthétique ou systémique dans lesquelles la vulnérabilité est perçue à la fois comme un risque biophysique et une réponse sociale (Cutter et al., 2003).

1) Un premier ensemble de définitions de la vulnérabilité relève des sciences physiques et ingénieuriales au sein desquelles le risque est pensé en termes de probabi-

9. Définitions données respectivement par Le Petit Larousse illustré, 2005, et Le Petit Robert : dictionnaire alphabétique et analogique de la langue française, 2004.

10. Il existe même un site internet dédié à ce concept : www.vulnerabilitynet.org/definitions. lité d'occurrence et d'impacts. La vulnérabilité renvoie alors au degré d'endommagement potentiel des enjeux suite à la réalisation d'un aléa dont les paramètres sont plus ou moins bien connus. Cette vulnérabilité « technique » ou «biophysique » est définie comme la conséquence en termes d'endommagement physique d'un aléa sur des enjeux (bâtiments, réseaux, infrastructures, populations...). Cela présente l'avantage de rendre la notion facile à quantifier, car ne sont pris en compte que des éléments matériels objectifs tels que l'intensité de l'aléa, le degré d'exposition, la densité de population, ou la qualité et la capacité de résistance des constructions (liée à la qualité des matériaux utilisés)... La lutte contre ce type de vulnérabilité en milieu urbain se traduit notamment par l'édiction de normes de construction, de réglementations et de planifications pour l'occupation des sols, ou par la mise au point de nouveaux matériaux et de nouvelles techniques de construction. Une telle définition de la vulnérabilité est critiquable dans la mesure où la société y est considérée comme passive, victime de la défaillance des systèmes bâtis ou techniques. Or, la réalité du risque est bien plus complexe. Elle fait intervenir de nombreux autres paramètres (notamment sociaux, culturels, ou économiques), qui doivent également être pris en compte, ce que tentent de faire les deux autres types de définitions.

2) Un deuxième ensemble regroupe les définitions de la vulnérabilité employées en sciences humaines et sociales qui ont fait évoluer ce concept en fonction du constat suivant : l'impact de l'aléa ne dépend pas seulement de l'exposition à la source de danger, mais est également conditionné par les propriétés sociales intrinsèques du territoire. Ces approches, moins aléa-centrées, cherchent à déterminer les conditions de l'endommagement et, par extension, la capacité de réponse de l'entité menacée (Veyret et Reghezza, 2006). La vulnérabilité désigne alors l'absence de capacité de la société à faire face à une crise ou à un changement, la difficulté d'une personne, d'un groupe d'humains, d'une organisation ou d'un territoire, à anticiper un phénomène destructeur, à l'affronter, à lui résister et à récupérer après sa survenue. Ces définitions perçoivent cette vulnérabilité socialement construite comme un état antérieur à la catastrophe, une propriété de l'enjeu considéré dépendant d'un certain nombre de facteurs structurels, qui se révèle lors de l'occurrence d'un aléa (CapHaz-Net, 2010).

Au fil de ses prolongements, la vulnérabilité appréhendée dans sa dimension "sociale ${ }^{11}$ » n’a plus été uniquement perçue comme la perte potentielle de choses inertes, mais est devenue une caractéristique des

11. Dans le cas des systèmes humains, on emploie de préférence le terme de "vulnérabilité sociale » tandis que, pour tout autre type de système (notamment un écosystème), on parle de « vulnérabilité inhérente»(Brooks, 2003, p. 4). 
groupes sociaux agissants qui contribuent eux-mêmes à «produire» le risque. En d'autres termes, la vulnérabilité n'est plus entendue « seulement comme l'ensemble des dommages potentiels provoqués lorsqu'un aléa se réalise, mais également comme l'ensemble des facteurs qui rendent une société plus ou moins fragile face à des événements désastreux »(Revet, 2008). Appliquée à la ville ou aux territoires, le concept de vulnérabilité sociale, qui fonde leur capacité de résilience, est ainsi bien plus large que la seule propension à ce que les constructions s'effondrent et à ce que les infrastructures soient endommagées ; selon Cannon et al. (2003), elle renvoie à une série de caractéristiques liées aux personnes, aux communautés et à leur environnement qui incluent le bien-être initial (statut nutritionnel, santé physique et mentale), les moyens d'existence (ressources et capitaux, revenus et qualifications) et le niveau de développement (taux d'urbanisation, taux de croissance et de vitalité économique...), l'autoprotection (capacités et volonté de construire une habitation sûre et d'utiliser un site sûr), la protection sociale (mesure de préparation et d'atténuation), les réseaux sociaux et politiques, ou encore les institutions (capital social, environnement institutionnel et les proches). La vulnérabilité sociale découle ainsi principalement de facteurs sous-jacents ${ }^{12}$ tels que des moyens d'existence précaires, des degrés d'autoprotection ou de protection sociale insuffisants, de dysfonctionnements ou d'inadéquations des installations institutionnelles, qui accroissent les inégalités sociales (de revenu, d'âge, de genre, etc.) ou écologiques (d'exposition à des nuisances ou divers facteurs de risques, etc.) et définissent le contexte global au sein duquel un individu ou une communauté endure et répond aux impacts négatifs d'un événement aléatoire (Cutter et al., 2003). Ces facteurs de vulnérabilité sociale, très souvent liés et imbriqués, interagissent entre eux (agir sur un facteur peut avoir des conséquences sur un autre, etc.), et sont difficilement chiffrables et quantifiables.

Alors que la vulnérabilité biophysique est une vulnérabilité spécifique à l'aléa, la vulnérabilité sociale, multidimensionnelle, ne l'est pas forcément : cette dernière est une propriété du système qui existe indépendamment de l'aléa au sens où un système est vulnérable que l'aléa se produise ou non. Elle est en revanche latente en ce sens que seule l'occurrence de l'aléa la révèle et lui permet de se manifester. L'impact de l'aléa ne dépend donc pas seulement de son intensité ou de l'exposition des enjeux à la source de danger : il est conditionné par les propriétés sociales du système affecté qui déterminent la sensibilité

12. Dans le cadre de la vulnérabilité sociale, dépendante de la façon dont la société est organisée et organise le territoire, ce sont plutôt les causes secondaires ou indirectes des dommages potentiels qui sont recherchées dans les propriétés sociales et intrinsèques du système. et les capacités adaptatives des enjeux face aux aléas. Par conséquent, « les lieux vulnérables du point de vue biophysique ne sont pas toujours ceux des populations vulnérables » (Ford, 2002, p. 14). Ford rappelle ainsi que, dans certaines régions exposées à un aléa intense, les pertes économiques pourront être élevées, mais du fait des systèmes d'assurance ou d'aide à la reconstruction, les populations pourront se relever rapidement. De la même façon, un système de gestion de crise efficace, des infrastructures de prise en charge des victimes performantes, une protection civile bien équipée et bien formée permettront de limiter le nombre de victimes.

D'Ercole et Thouret (1996) ont proposé une synthèse classifiant les divers facteurs de vulnérabilité sociale. Ces facteurs peuvent être d'ordre structurel ou conjoncturel (même si la plupart du temps, les facteurs conjoncturels sont rattachés à des facteurs structurels plus profonds), ou encore être génériques ou spécifiques. Les facteurs structurels permanents, dont l'évolution rend une population et ses biens plus ou moins vulnérables, sont multiples : sociodémographiques et économiques ; socioculturels (degré de connaissance et d'information sur le risque, expérience) ; techniques ou physiques, liés au bâti et aux matériaux exposés ; fonctionnels, désignant la qualité de la prévention et de l'organisation des secours en cas de catastrophe ; enfin, institutionnels ou politico-administratifs (implication, coordination des acteurs compétents, organisation de la gestion de crise, de la reconstruction), fondant la planification et la réglementation en matière de risque. En outre, d'autres facteurs, géographiques et conjoncturels, rendent compte d'une vulnérabilité contingente, c'est-à-dire temporaire

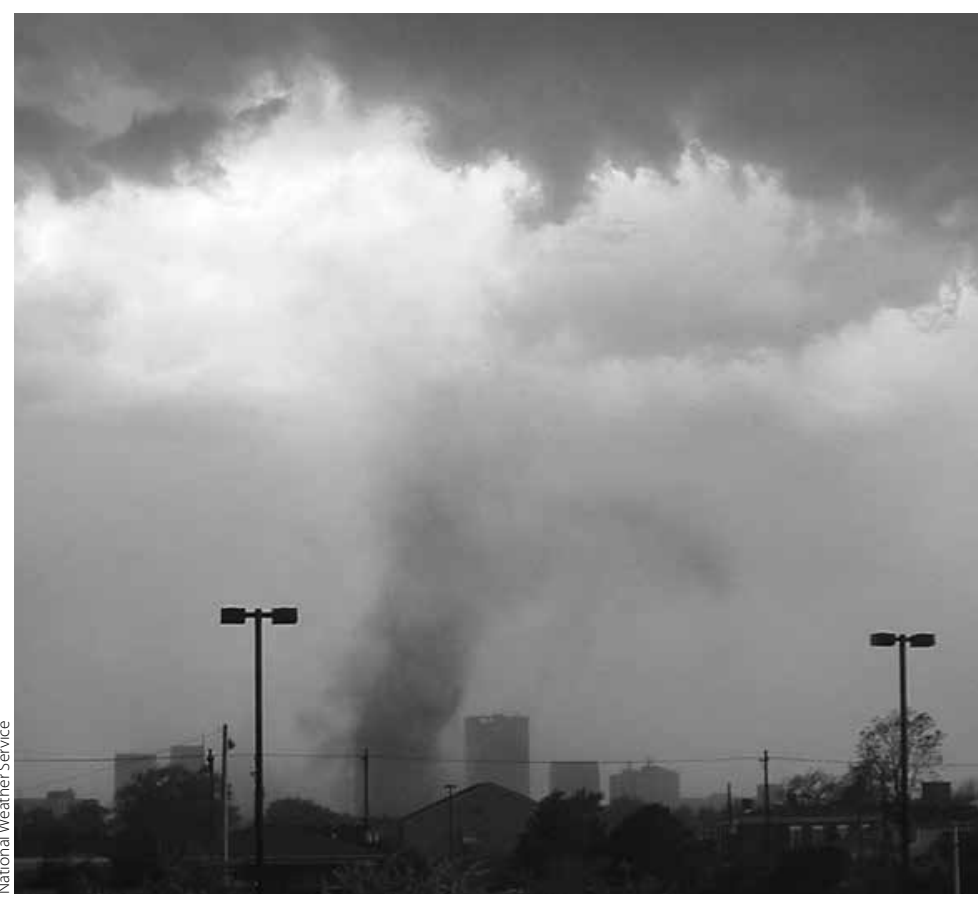


et imprévisible, dans un lieu donné et au moment de l'impact d'un aléa. Parmi ces facteurs, certains sont dits "génériques », au sens où ils déterminent une vulnérabilité valable pour tous les aléas. D'autres sont en revanche "spécifiques ", au sens où ils conditionnent uniquement la capacité de résistance à certains types d'aléa. Les facteurs purement sociaux, structurels, sont la plupart du temps des facteurs génériques. Ils influent en revanche sur d'autres facteurs, notamment sur les propriétés physiques de résistance, qui eux sont spécifiques ${ }^{13}$ (D'Ercole et Thouret, 1996).

Cependant, une définition commune fait défaut à ce concept de vulnérabilité sociale (Blaikie et al., 1994 ; Wisner et al., 2004), ce qui signifie que les différents auteurs la conçoivent et l'utilisent de diverses manières (CapHaz-Net, 2010). Suivant les auteurs, la vulnérabilité « sociale » peut ou non inclure les éléments de la vulnérabilité « biophysique ». Ainsi, pour Cutter et al. (2003), la vulnérabilité sociale englobe davantage que les impacts socio-économiques, puisqu'elle comprend également les caractéristiques des dommages physiques potentiels dans l'environnement construit ; dans une perspective proche, Downing et al. (2006) définissent six attributs qui caractérisent la vulnérabilité sociale : une exposition différenciée aux expériences de stress ou anticipées par les différentes unités exposées; un processus dynamique ; un enracinement dans les actions et les multiples attributs des acteurs humains; une détermination fréquente par les réseaux sociaux dans les interactions sociales, économiques, politiques et environnementales ; une manifestation simultanée sur plusieurs échelles; et enfin, une influence et une émergence provoquée par de multiples stress. En revanche, d'autres auteurs distinguent clairement les fragilités socio-économiques et le manque de résilience en tant que conditions du contexte social (qui favorisent les impacts de second ordre), d'une part, et les dommages physiques causés par l'exposition et la sensibilité physique de l'environnement construit (reliés aux impacts de premier ordre), d'autre part (Cardona, 2004). Reste que la «somme » des deux vulnérabilités « technique ou biophysique » et «sociale » permet de représenter la «vulnérabilité globale » du territoire exposé à l'aléa (Revet, 2008).

3) Un troisième ensemble relève des approches systémiques qui tentent de faire la « synthèse » entre les vulnérabilités biophysiques et sociales au sein d'un système unique qualifié de système socio-écologique (SSE) complexe. La plupart des auteurs qui se rattachent à cette vision soulignent le fait que la vulnérabilité glo-

13. La pauvreté peut, par exemple, être responsable du non-respect des règles de construction ou de l'emploi de matériaux inadaptés conduisant à ce que les bâtiments soient alors vulnérables aux séismes, mais pas à la sécheresse. bale (biophysique et sociale), non plus entendue comme un état mais comme un processus, est simultanément multidimensionnelle et différentielle (elle varie suivant les espaces physiques et entre et au sein des groupes sociaux) ; dépendante des échelles spatio-temporelles (elle varie aussi dans le temps et dans l'espace) et des unités d'analyse retenues (individus, ménages, groupes ou communautés, régions, éléments ou systèmes, etc.) ; dynamique (les caractéristiques et les moteurs de la vulnérabilité évoluent dans le temps). L'approche systémique de la vulnérabilité globale comprend ainsi diverses caractéristiques qui sont gouvernées par de multiples stress, des expositions et des sensibilités différenciées et sont souvent enracinées dans de multiples attributs et circonstances de l'environnement élargi (y compris liées aux acteurs humains et réseaux sociaux), qui rendent les personnes ou les communautés aptes à faire face aux impacts d'un événement aléatoire, ou qui, à l'inverse, limitent leur aptitude à y résister ou à s'y adapter (ce qui renvoie à leur résilience). Cela souligne aussi le fait que l'analyse des trajectoires de dommages peut contribuer à l'identification des vulnérabilités révélées comme à l'estimation des vulnérabilités actuelles et potentielles dans le futur. Le défi réside alors dans la possibilité de développer une approche équilibrée entre le contexte général (indicateurs macro-économiques) d'un côté, et le contexte local (indicateurs plus précis et spécifiques, soit locaux, soit relevant des méso-échelles) et qui puisse aussi s’appuyer sur les vulnérabilités révélées dans le passé.

\section{Les multiples facettes de la vulnérabilité} dans les approches des risques « naturels »

L'un des enseignements que l'on peut tirer des développements ci-dessus relatifs à la vulnérabilité est qu'au fur et à mesure de l'approfondissement des analyses sur cette question, on a assisté à une complexification et un élargissement progressif du concept (Birkmann, 2005), suivant un continuum de définitions allant de la plus étroite (vulnérabilité intrinsèque définie comme un facteur de risque interne) à la plus large (vulnérabilité multidimensionnelle englobant des caractéristiques physiques, sociales, économiques, environnementales et institutionnelles) (processus d'élargissement symbolisé par la flèche partant de la sphère la plus étroite et allant vers la plus large dans la figure page suivante).

Quasiment toutes les conceptions de la vulnérabilité ont en commun de la percevoir comme la « face interne du risque ", en lien étroit avec les discussions autour de la vulnérabilité comme caractéristique intrinsèque d'un système ou d'un élément exposé à un risque. Cela signifie que les conditions de l'unité ou de la communauté (sensibilité) exposées à un risque sont perçues comme les caractéristiques centrales de la vulnérabilité (UN/ISDR, 2004 ; 
Cardona, 2004 ; Wisner, 2002), et représentent le socle commun de toutes les définitions avancées de la vulnérabilité (illustré par le plus étroit des cercles situé au cœur de la figure ci-dessous).

\section{Les sphères-clés du concept de vulnérabilité}

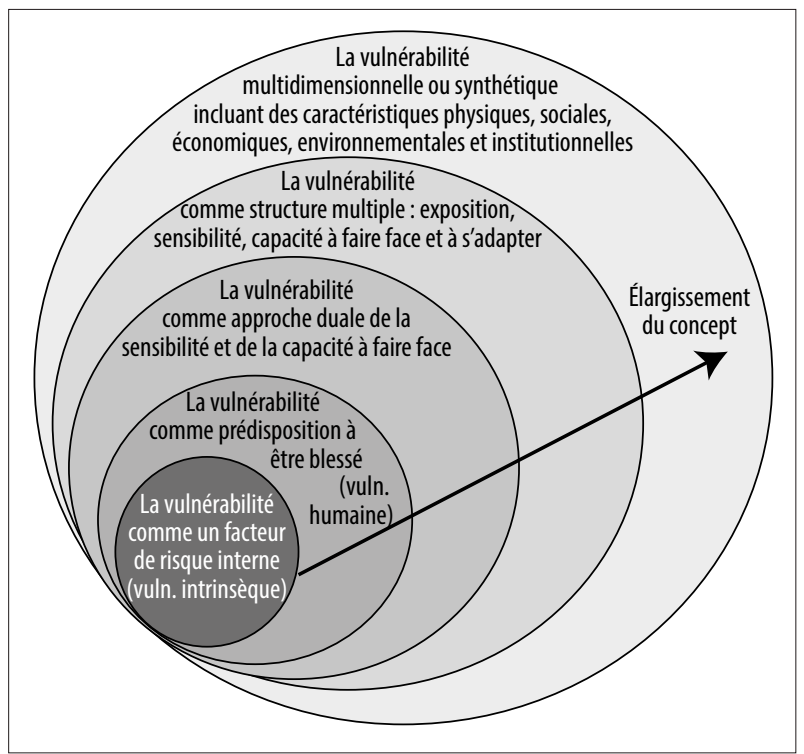

Source : d'après Birkmann (2005).

De manière intéressante, cette compréhension de la vulnérabilité comme la «face interne du risque » et comme une " caractéristique intrinsèque » d'un élément soumis au risque peut s'appliquer à des entités très diverses, telles que des communautés, des groupes sociaux (conditions socio-économiques, cadre institutionnel), des structures et des caractéristiques physiques de constructions et/ou de lignes de vie (structure physique), de même qu'à des écosystèmes ou des fonctions et des services environnementaux (écosystèmes, capital environnemental).

Une première extension de cette définition peut être perçue au travers de celle avancée par Wisner (2002), qui définit la vulnérabilité, principalement reliée aux personnes, comme désignant la prédisposition à souffrir de blessures, décès, pertes et ruptures dans les moyens d'existence face à un événement extrême et/ou de difficultés inhabituelles à récupérer des impacts négatifs provoqués par des aléas. Cette définition souligne le fait que les éléments principaux de la vulnérabilité sont constitués par les conditions qui accroissent et déterminent la prédisposition des êtres humains à subir des dommages. Par conséquent, une deuxième sphère, plus large et englobant la première, peut être dessinée à partir de cette définition anthropocentrée.

Sur la base du deuxième cercle, une nouvelle extension de la vulnérabilité a été opérée : la " prédisposition à être blessé » a en effet été élargie par l'accent mis sur une structure dualiste de la vulnérabilité qui peut être observée dans les définitions proposées par Wisner (2002) et partiellement par Bohle (2001). Wisner identifie clairement la « prédisposition à la blessure » et les " difficultés inhabituelles à récupérer » face à de tels événements comme étant les caractéristiques clés de la vulnérabilité. Cela signifie que le concept de vulnérabilité est élargi en percevant celle-ci comme impliquant une approche à double face, avec, d'un côté, la sensibilité et, de l'autre, les difficultés inhabituelles à faire face et à se remettre du choc subi. Cependant, la double structure de la vulnérabilité mise en avant par Bohle (2001) ne consiste pas simplement à distinguer l'« exposition » et la " capacité à faire face »; cette dualité réside plutôt dans la séparation entre les caractéristiques externes (l'exposition aux risques et aux chocs) et internes (capacité à anticiper et à faire face aux impacts d'un aléa) de la vulnérabilité d'un élément exposé à un risque. Dans ce contexte, une troisième sphère peut être associée à cette vision duale de la vulnérabilité, qui souligne le fait que la vulnérabilité est formée et déterminée par la sensibilité comme propension à souffrir d'une blessure (connotation négative) et par l'aptitude et la capacité à faire face et à récupérer (connotation positive) de ces stress et impacts négatifs liées à l'occurrence de l'aléa (Wisner, 2002).

À partir de cette troisième sphère, le passage d'une conception à « double structure " à une «structure multidimensionnelle » de la vulnérabilité peut être interprété lui aussi comme un élargissement additionnel du concept (figure à gauche). L'appréhension de la vulnérabilité dans la communauté scientifique sur le changement climatique ou au sein de celle du développement durable met ainsi en lumière le fait que ce concept englobe non seulement l'exposition, la sensibilité et la capacité à faire face, mais également les capacités adaptatives, les réponses (adaptation) et les interactions avec les perturbations et les stress. Cela permet de tracer une quatrième sphère, qui étend encore davantage le concept de vulnérabilité conçue comme une structure multidimensionnelle complexe en interaction avec les aléas. À cet égard, alors que la perspective traditionnelle de l'ingénierie des risques se concentre surtout sur les aspects (bio)physiques de la vulnérabilité, les approches plus systémiques mettent en avant la nécessité de prendre en compte différents paramètres qui forment et déterminent la vulnérabilité d'une communauté et de son territoire (UN/ISDR, 2004), telles que les caractéristiques physiques, économiques, sociales, environnementales et institutionnelles ; certaines approches soulignent aussi la nécessité d'intégrer des facteurs moteurs planétaires qui ont un impact sur la vulnérabilité, comme la mondialisation, l'urbanisation ou le changement climatique. Cela implique que l'attention se détourne d'une analyse aléa-dépendante fondée principalement sur la structure biophysique de la vulnérabilité pour s'orienter vers une analyse interdisciplinaire large et englobante du concept multidimensionnel de vulnérabilité (Cardona, 2004). 
La nature multidimensionnelle ou multifacettes (physique, systémique, sociale, économique, territoriale, institutionnelle, organisationnelle, et culturelle) de la vulnérabilité aux aléas a été symbolisée par l'analogie du diamant (Parker et al., 2009), sans que la taille et la position des différentes facettes ne soient représentatives de caractéristiques particulières ; le nombre de facettes et leurs interrelations sont encore fortement débattues au sein de la littérature ; la nature et parfois même la direction des relations entre chaque facette n'a pas encore été clarifiée, bien que l'analogie du diamant reconnaisse que de telles relations existent. La force de ces relations et les liens entre les différentes dimensions de la vulnérabilité varient d'une facette à l'autre, de même que dans le temps et l'espace (figure ci-dessous).

\section{Analogie du diamant et multiples facettes de la vulnérabilité}

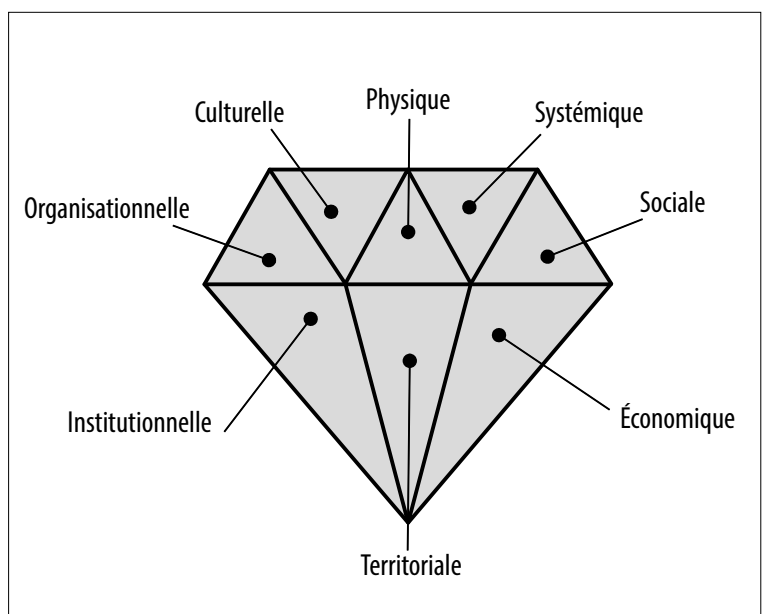

Source : Parker et al., (2009)

Dans le diagramme de l'analogie du diamant, la vulnérabilité «physique » ou «structurelle » est perçue comme la potentialité de dommages structurels sur les constructions, les véhicules, les infrastructures et les « lignes de vies » (chauffage, nourriture, eau) et peut éventuellement inclure les faiblesses physiques des humains ${ }^{14}$. Elle traduit le niveau de protection que les aménagements ou les ouvrages assurent aux enjeux exposés (personnes, biens et activités) pour partie liée à leur conception architecturale et technique (Guézo et Verrhiest, 2006). Elle se rapproche de la vulnérabilité géographique résultant des caractéristiques physiques d'un territoire qui entrent en résonance avec celles de l'aléa et qui correspond à l'exposition d'un

14. La fragilité « physique » d'une personne confrontée à une crise majeure recouvre la vulnérabilité intrinsèque à la personne (capacité personnelle à résister à un aléa donné et à réagir face à la catastrophe), mais aussi son exposition au phénomène dangereux lorsqu'il survient. territoire et des enjeux au phénomène dangereux ${ }^{15}$, quelle que soit l'origine de l'aléa. La vulnérabilité « systémique » est associée à l'endroit et à la manière dont un événement pourrait se propager au travers de systèmes et leur susceptibilité d'être inaptes à fonctionner (i.e. les lignes de vie). Elle résulte des effets différés ou induits par l'occurrence d'un aléa, effets dus aux proximités, aux interactions et interdépendances, qui se nouent entre les occupations de l'espace, les usages, les activités et les enjeux exposés, que ce soit en interne au système considéré (urbain par exemple) ou en relation avec lui ${ }^{16}$. La vulnérabilité « culturelle» renvoie à la potentialité de pertes en croyances indigènes, coutumes ou habitudes, et en artefacts et modes de vie qui leur sont liés. Cela peut inclure l'indépendance culturelle et la surimposition d'idées et de concepts provenant de sources externes. La vulnérabilité " organisationnelle » se réfère à la potentialité de pertes et d'une aptitude réduite à récupérer causées par l'exposition d'individus, de communautés ou d'économies locales aux conséquences néfastes découlant de défauts cruciaux d'une organisation ${ }^{17}$. La vulnérabilité « institutionnelle » élargit l'attention sur les arrangements institutionnels et les conséquences potentielles de défauts critiques des institutions et des arrangements institutionnels. La vulnérabilité « territoriale »,

15. Dans le cas d'une inondation, par exemple, les conditions d'écoulement des eaux en lien avec la topographie et l'occupation du sol déterminent les dommages. Tous les enjeux exposés ne présentent pas la même vulnérabilité géographique à un aléa donné ainsi, les constructions situées en zone inondable sont différemment menacées ; celles situées sur des promontoires peuvent même être hors d'atteinte des eaux les plus hautes (Guézo et Verrhiest, 2006).

16. La vulnérabilité systémique ou de dépendance est associée à l'endroit et à la manière dont un événement pourrait se propager au travers des sous-systèmes urbains (i.e. les lignes de vie) rendus inaptes à fonctionner ou amenés à fonctionner en mode dégradé. Les trois exemples ci-après illustrent cette vulnérabilité : une coupure d'électricité perturbe, par ses effets directs, le fonctionnement urbain, mais elle peut également avoir des effets induits tels que l'interruption de l'alimentation en eau potable ; la sécurité d'une agglomération ou d'un quartier est indirectement tributaire de la qualité de la gestion de la sécurité dans les établissements à risque ou d'ouvrages tels que les barrages ou les digues; l'arrêt d'une activité économique lié à des défauts d'approvisionnement occasionnés par l'inondation (dommages chez le fournisseur ou coupure des voies de circulation) augmente de façon significative le coût du sinistre (Guézo et Verrhiest, 2006).

17. La vulnérabilité organisationnelle d'une ville dépend de son niveau de préparation face aux aléas (plans de secours et d'intervention d'urgence, plans d'évacuation et de déplacements, système de surveillance, d'alerte et de communication, etc.) pour limiter les effets d'un événement catastrophique sur ses enjeux (services publics en réseaux et administratifs, établissements recevant du public, centres stratégiques, entreprises, institutions et arrangements institutionnels...), et pour rétablir rapidement des conditions acceptables de fonctionnement. Pour une entreprise, la localisation de biens de valeur dans des locaux inondables sans possibilité de pouvoir les déplacer en cas de besoin traduit une vulnérabilité organisationnelle alors qu'un aménagement intérieur adéquat éviterait des pertes matérielles (Guézo et Verrhiest, 2006). 
un concept fortement débattu au sein des sciences sociales et politiques, implique une unité d'espace et une territorialité ${ }^{18}$. Cette dernière est vue par Parker et al. (2009) comme un assemblage unique et dynamique de culture, de croyances historiques et actuelles, d'habitudes, d'attitudes, d'actifs et de capitaux qui caractérisent, ou même définissent, un espace ou un territoire spécifique et ses liens avec les autres territoires. La vulnérabilité « économique » est perçue comme la potentialité de pertes en actifs économiques et en productivité, y compris la perte de moyens d'existence que ces actifs supportent, la perte de richesse et d'indépendance économique qu'ils créent ensemble avec la perte financière, la dépendance à la dette et l'incapacité à récupérer des pertes. Enfin, la vulnérabilité "sociale ", pour laquelle existent une multitude de définitions (voir supra), est elle-même multidimensionnelle. L'absence de culture du risque, de politique de gestion, ou de mécanismes d'aide à la reconstruction... sont autant de facteurs de vulnérabilité sociale eux-mêmes déterminés par les facteurs sous-jacents de la capacité adaptative (Guézo et Verrhiest, 2006).

Au sein des différentes approches de la vulnérabilité, celles qui apparaissent les plus prometteuses pour envisager la vulnérabilité urbaine aux risques climatiques actuels et futurs reconnaissent qu'elle relève d'une dynamique complexe, non linéaire (Quenault, 2014). En principe, la vulnérabilité augmente avec l'exposition aux aléas climatiques et la sensibilité du système urbain à leurs impacts, tandis qu'elle diminue à mesure que sa capacité adaptative s'accroît. Les événements dangereux quand ils surviennent révèlent les vulnérabilités existantes de même qu'ils peuvent créer les vulnérabilités futures en renforçant la sensibilité du système urbain à l'égard des effets néfastes de futurs aléas. Toutefois, la réalité est plus complexe que cette simple causalité linéaire, la vulnérabilité se trouvant au cœur de plusieurs boucles de rétroactions (tant positives que négatives) caractéristiques d'une causalité circulaire (Quenault et al., 2011 ; Quenault, 2013b).

Le passage de la notion de vulnérabilité du champ de la recherche à celui de l'action, du statut de catégorie d'analyse à celui de catégorie pratique par le biais de politiques publiques ou de programmes mis en place par des acteurs privés afin de l'enrayer, n'est pas sans conséquences. En permettant de définir des situations dans lesquelles des personnes ou des groupes, des infrastructures ou des territoires sont jugés menacés et incapables de faire face ou de répondre à un danger ou un aléa, la notion de

18. En géographie, le concept de territoire, qui se désigne par son nom, se définit par des limites et est associé à des acteurs, s'entend comme un espace sur lequel s'exercent un ou des pouvoirs. Or, la gestion du risque repose sur la territorialisation de ce dernier et, assigner un territoire au risque, suppose de le circonscrire spatialement (Barbier et Larrue, 2011). vulnérabilité appelle des interventions dont la justification est contenue dans la notion elle-même, avec un résultat sous forme de paradoxe : " davantage de connaissances et davantage de dégâts » (Weichselgartner, 2004, p. 212). Comment expliquer cet apparent paradoxe suivant lequel toujours plus de gestion préventive des risques s'accompagne pourtant de toujours plus de catastrophes? Le paradoxe, apparemment lié au décalage entre l'affirmation officielle de la protection accrue face aux aléas et l'augmentation des dommages que celle-ci favorise, permet de comprendre que les politiques gérant les risques sont forcément imparfaites, en ce sens qu'elles produiront nécessairement des effets non désirés, inattendus (Quenault et al., 2011). Le paradoxe disparaît ainsi dès lors que survient la reconnaissance du fait que gestion des risques climatiques et urbanisation coévoluent : la gestion préventive des catastrophes favorise l'urbanisation qui ne cesse de se recomposer en s'intensifiant précisément parce qu'elle ne permet jamais d'éliminer totalement les risques compte tenu des limites intrinsèques des politiques supposées les gérer (Pigeon, 2010). La vulnérabilité urbaine ne désigne donc pas seulement le fait que la ville puisse être plus ou moins à même de subir des dommages dus aux aléas climatiques : « La vulnérabilité urbaine est aussi la capacité des villes et de leurs habitants à engendrer les conditions de la survenue de ces phénomènes ou du moins à en aggraver les effets » (D'Ercole et al., 2009, p. 41 1). Force est en effet de constater que l'accroissement des risques et catastrophes d'origine climatique en milieu urbain a « une double dimension anthropique : il est lié à l'anthropisation des aléas (inondations par ruissellement urbain, par exemple) et à l'accroissement de la vulnérabilité des sociétés " (Beccera et Peltier, 2009, p. 6). Si l'origine de ces catastrophes est « un assemblage inextricable de faits sociaux et d'états de nature »(Cronon, 1996), elle tient aussi à une chaîne d'interactions complexes entre urbanisation et phénomènes météorologiques, les villes traversant une profonde mutation sous l'emprise de changements climatiques, démographiques et environnementaux. Il convient en effet de reconnaître l'intrication des tendances de fonds qui transforment l'environnement des sociétés humaines et les mettent au défi de pouvoir se protéger face aux effets de multiples risques transversaux et interdépendants qui ne sont pas uniquement liés au changement climatique ; ces interactions induisent des dynamiques complexes marquées par des surprises, des effets en retour, des seuils critiques, des bifurcations (Quenault, 2014).

Les réponses envisagées face à la menace climatique ne peuvent se cantonner au traitement d'un événement spécifique car une crise peut toucher les systèmes de manière globale et polymorphe (procédures habituelles hors-jeu ; insuffisances d'informations pour décider). Ces considérations militent pour une approche radicalement interdisciplinaire des aléas climatiques et de leurs effets en contexte urbain afin de pouvoir mieux comprendre 
les situations de crise générées par les vulnérabilités et leur inscription dans la temporalité plus longue de la gestion et de la planification urbaines (Barles, 2010). Par conséquent, les risques futurs de catastrophe associés au changement climatique seront, dans une large mesure, déterminés par le mode de gestion et de planification des villes et des métropoles dont les vulnérabilités sont de plus en plus complexes en lien avec l'interconnexion et l'interdépendance croissantes des sociétés modernes en proie à la mondialisation (Quenault, $2012 ; 2015$ ).

\section{Références bibliographiques}

Adam (Adaptation and Mitigation Strategies: Supporting European Climate Policy), (2008), D-A1.1: A formal framework of Vulnerability, Project $n^{\circ} 018476$ GOCE, avril.

Adger W. N., (2006), «Vulnerability », Global Environmental Change, vol. 16, n³, pp. 268-281.

Barbier R., Larrue C., (2011), « Démocratie environnementale et territoires : un bilan d'étape», Participations, $n^{\circ} 1$, pp. 67-104

Barles S., (2010), « Systèmes urbains et événements climatiques extrêmes », in Décamps H. (dir.), Evvénements climatiques extrêmes: réduire les vulnérabilités des systèmes écologiques et sociaux, Rapport sur la science et la technologie, $n^{\circ} 29$, Paris, Académie des Sciences, Éditions EDP Sciences, juin, pp. 67-72.

Beccera S., Peltier A., (2009), «La vulnérabilité sociétale aux risques naturels et aux problèmes environnementaux: comprendre pour réduire. Avant Propos », in Beccera S. et Peltier A. (dir.), Risques et environnement: recherches interdisciplinaires sur la vulnérabilité des sociétés, Paris, L'Harmattan (Sociologies et environnement), pp. 5-8.

Beck U., ([1986] 2001), La société du risque, Paris, Aubier (Alto).

Blaikie P., Cannon T., Davis I., Wisner B., (1994), At Risk - Natural hazards, people's vulnerability and disasters, London/New York, Routledge.

Birkmann J., (2005), « Danger Need Not Spell Disaster. But How Vulnerable Are We? », Research Brief, (1), Tokyo, United Nations University.
Bohle H.-G., (2001), «Vulnerability and Criticality: Perspectives from Social Geography », IHDP Update 2/2001, Newsletter of the International Human Dimensions Programme on Global Environmental Change, pp. 1-7.

Brooks N., (2003), « Vulnerability, risk and adaptation: A conceptual framework », Working Paper, $n^{\circ} 38$ sept., Tyndall Centre for Climate Change Research, [en ligne] http://www.tyndall.ac.uk/sites/default/files/ wp38.pdf.

Calvo C., Dercon S., (2005), « Measuring Individual Vulnerability », Department of Economics Discussion Paper Series, 229, [en ligne] http://ideas.repec.org/p/ oxf/wpaper/229.html.

Cannon T., Twigg J., Rowell J., (2003), Social Vulnerability. Sustainable Livelihoods and Disasters, Report to DFID Conflict and Humanitarian Assistance Department (Chad) and Sustainable Livelihoods Support Office, [en ligne] www.benfieldhrc.org/ disaster_studies/projects/soc_vuln_sust_live.pdf.

CapHaz-Net, 2010, Social Capacity Building for Natural Hazards, Toward More Resilient Societies, WP4 Report, [en ligne] http://www.caphaz.net.org.

Cardona O. D., (2004), «The Need for Rethinking the Concepts of Vulnerability and Risk from a Holistic Perspective: A Necessary Review and Criticism for Effective Risk Management », in Bankoff G., Frerks G. et Hilhorst D. (Éds.), Mapping Vulnerability: Disasters, Development and People, London, Earthscan, chap. 3.

Cred (Centre for Research on the Epidemiology of Disasters), (2014), Annual Disaster Statistical Review 2013. The numbers and trends, Cred, université catholique de Louvain, Brussels, [en ligne] http://cred. be/download/download.php?file=sites/default/files/ ADSR_2013.pdf.
Cred (Centre for Research on the Epidemiology of Disasters), (2010), EM-DAT, the Ofda/Cred International Disaster Database, Bruxelles, université Catholique de Louvain, [en ligne] www.emdat.be/Database/Trends/ trends.html.

Cronon W., (1996), Uncommon ground: Rethinking the Human Place in Nature, New York, W.W. Norton and Company.

Cutter S. L., Boruff B. J., Shirley W. L., (2003), « Socia Vulnerability to Environmental Hazards », Social Science Quarterly, vol. 84, n² 2, juin, pp. 242-261.

D’Ercole R., (1994), « Les vulnérabilités des sociétés et des espaces urbanisés : concepts, typologie, modèles d'analyse », Revue de Géographie Alpine, vol. 82, n 4, pp. 87-96.

D’Ercole R., Hardy S., Metzger P., Robert J., (2009), «Vulnérabilités urbaines dans les pays andins, Introduction générale », in D'Ercole R. et al. (dir.), Vulnerabilidades urbanas en los paises andinos (Bolivia Ecuador, Perú). Bulletin de l'Institut français d'études andines, t. 38, n³ Lima, Ifea, pp. 411-420.

D’Ercole R., Metzger P.» (2009), « La vulnérabilité territoriale : une nouvelle approche des risques en milieu urbain », Cybergeo: European Journal of Geography, Dossier Vulnérabilités urbaines au Sud, art. 447, [en ligne] http://cybergeo.revues.org/22022.

D’Ercole R., Thouret J.-C. (1996), «Vulnérabilité aux risques naturels en milieu urbain : effets, facteurs et réponses sociales », Cahier des sciences humaines, vol. 32, n², pp. 407-422, [en ligne) http://horizon. documentation.ird.fr/exl-doc/pleins_textes/pleins_ textes_4/sci_hum/010006241.pdf. 


\section{Références bibliographiques}

Downing T. E. et al., (2006), « Integrating social vulnerability into water management », SEI Working Paper and Newater Working Paper, $n^{\circ}$ 4, Oxford, Stockholm Environment Institute.

Dunglas J., (2005), «Typologie des aléas et connaissance scientifique de la vulnérabilité. Le point de vue de l'ingénieur », Les Annales des Mines, Responsabilité et Environnement. Les catastrophes naturelles, n²40, octobre, pp. 91-101.

Fabiani J.-L., Theys J., (1987), La société vulnérable: évaluer et maîtriser les risques, Paris, Presses de l'École normale supérieure.

Ford J., (2002), «Vulnerability: Concepts and Issues », Ph.D. Scholarly Field Paper, Guelph, University of Guelph.

Giec (Groupe intergouvernemental d'experts sur l'évolution du climat), (2007), «Bilan 2007 des changements climatiques : les bases scientifiques physiques, Groupe de travail I », in Quatrième rapport d'évaluation, 2 février, Paris, [en ligne] www.ipcc.ch

Gilbert C., (2006), «Préface », in Magne L. et Vasseur D. (dir.), Risques industriels. Complexité, incertitude et décision : une approche interdisciplinaire, Paris, Lavoisier/Éditions Tec et Doc, pp. 5-21.

Gray P. C. R., Wiedermann P. M., (1997), «Risk and Sustainability: Mutual Lessons from Approaches to the Use of Indicators (Second Edition) », [en ligne] www.fz-juelich.de/mut/hefte/heft_61.pdf.

Guézo B., Verrhiest G., (2006), « Dossier : Réduire la vulnérabilité urbaine aux risques majeurs », Techni. Cités, nº 108, avril.

Hewitt K., (1983), «The Idea of Calamity in the Technocratic Age », in Hewitt K., Interpretations of Calamity from the Viewpoint of the Human Ecology, London, Allen and Unwin.

O'Brien K., Eriksen S., Nygaard L., Schjolden A., (2007), «Why different interpretations of vulnerability matter in climate change discourses », Climate Policy, vol. 7, n 1, pp. 73-88.
Parker D. et al., (2009), « Deliverable 2.1. Relations between different types of social and economic vulnerability », Final draft report submitted to EU project Enhancing resilience of communities and territories facing natural and na-tech hazards (Ensure).

Pigeon P., (2010), « Catastrophes dites naturelles, risques et développement durable: Utilisations géographiques de la courbe de Farmer », VertigO. La revue électronique en sciences de l'environnement, vol. 10, n 1, avril, [en ligne] http://vertigo.revues. org/9491 (mis en ligne le 26/04/2010, consulté le 15/06/2013)

Pigeon P., (2005), Géographie critique des risques, Paris, Économica (Anthropos)

Quenault B., (2015), « La dialectique vulnérabilité/ adaptation des villes au changement climatique comme potentiel destructeur/créateur : quelles perspectives pour un développement urbain durable? », in Forest J. et Hamdouch A. (Éd.), Quand l'innovation fait la ville durable, Lausanne, Presses polytechniques universitaires romandes (Metis Lyon Tech), pp. 19-44.

Quenault B., (2014), « La résurgence/convergence du triptyque catastrophe-résilience-adaptation pour (re)penser la "fabrique urbaine" face aux risques climatiques », Développement Durable \& Territoires (DDT), vol. 5, n³ décembre, [en ligne], http:// developpementdurable.revues.org/10683 (mis en ligne le 5/12/2014)

Quenault B., (2013a), «Chapitre 6. La ville durable au défi du couple vulnérabilité/adaptation au changement climatique », in Bertrand F. et Rocher L. (Éds.), Les territoires face aux changements climatiques. Une première génération d'initiatives locales, P.I.E. Peter Lang, Ecopolis, n 18, Bruxelles, pp. 175-199.

Quenault B., (2013b), « Retour critique sur la mobilisation du concept de résilience en lien avec l'adaptation des systèmes urbains au changement climatique », EchoGéo, 24, [en ligne] http://echogeo. revues.org/13403 (mis en ligne le 10/07/2013).
Quenault, B., (2012), «Chapitre 11. Mondialisation, mutations urbaines et vulnérabilité au changement climatique : quelles stratégies de résilience pour un développement urbain durable ? », in Hamdouch A. Depret M.-H. et Tanguy C. (dir.), Mondialisation et résilience des territoires: trajectoires, dynamiques d'acteurs et expériences locales, Québec, Presses de I'université du Québec (Géographie contemporaine), pp. 175-199.

Quenault B. et al., (2011), Vulnérabilité et résilience au changement climatique en milieu urbain : vers des stratégies de développement urbain durable? projet de recherche Pirve 20-2051, programme interdisciplinaire Ville Environnement (Meddat/ CNRS), Maison des sciences de l'homme de Bretagne (MSHB), juin.

Revet S., (2008), «La vulnérabilité, une notion problématique? Un regard d'anthropologue », in actes du colloque Vulnérabilités sociétales risques et environnement: comprendre et évaluer, 14-16 mai, université Toulouse-Le Mirail, p. 1.

UN/ISDR (International Strategy for Disaster Reduction), (2004), Living with Risk: A Global Review of Disaster Reduction Initiatives, Geneva, UN Publications.

Veyret Y., Reghezza M., (2006), « Vulnérabilité et risque, l'approche récente de la vulnérabilité », Responsabilité et environnement, Les Annales des Mines, $n^{\circ}$ 43, juillet, pp. 9-13.

Weichselgartner J., (2004), « Changer au rythme des changements : les défis s'adressant à la gestion des risques naturels », in Veyret Y., Garry G. et Meschinet de Richemond N. (dir.), Risques naturels et aménagement en Europe, Paris, Armand Colin, pp. 212-222.

Wisner B. (2002), «Who? What? Where? When? In an Emergency: Notes on Possible Indicators of Vulnerability and Resilience: By Phase of the Disaster Management Cycle and Social Actor », in Plate E. (Éd.), Environment and Human Security, Contributions to a workshop in Bonn, 23-25 octobre, Germany, pp. 12/7-12/14

Wisner B. et al., (2004), At risk- natural hazards, people's vulnerability and disasters, London, Routledge. 


\section{Biographie}

BÉATRICE QUENAULT est maître de conférences en économie au sein du laboratoire Espaces et sociétés (ESO UMR 6590) de l'université Rennes 2. Ses recherches, centrées sur les questions liées aux enjeux du développement durable et du changement climatique s'inscrivent dans le cadre de l'économie politique de la mondialisation et de l'aménagement durable des territoires. Initialement focalisées sur les enjeux d'équité et de solidarité du développement durable et de la lutte concertée contre le changement climatique, appelant un nouveau système de gouvernance mondial et local, ses préoccupations de recherche portent désormais sur les questions de vulnérabilité, résilience et adaptation urbaine au changement climatique. Elle a notamment publié, en 2015, «La dialectique vulnérabilité/adaptation des villes au changement climatique comme potentiel destructeur/créateur : quelles perspectives pour un développement urbain durable? », in Forest J. et Hamdouch A. (Éd.), Quand l'innovation fait la ville durable, Lausanne, Presses polytechniques universitaires romandes (Metis Lyon Tech), pp. 19-44 ; en 2014, « La résurgence/ convergence du triptyque catastrophe-résilienceadaptation pour (re)penser la "fabrique urbaine" face aux risques climatiques », Développement Durable \& Territoires (DDT), vol. 5, n³, décembre, [en ligne], http://developpementdurable.revues.org/10683 (mis en ligne le 5/12/2014).

beatrice.quenault@univ-rennes2.fr 